\title{
Laser arrival measurement tools for SwissFEL
}

\author{
Marta 'Csatari' Divall, ${ }^{* a}$ Albert Romann, ${ }^{a}$ Patrick Mutter, ${ }^{a}$ Stephan Hunziker, ${ }^{a}$ Christoph P. Hauri ${ }^{\mathrm{a}, \mathrm{b}}$ \\ ${ }^{\mathrm{a} P a u l}$ Scherrer Institut, 5232 Villigen PSI, Switzerland; ${ }^{\mathrm{b}}$ Ecole Polytechnique Fédérale de Lausanne, \\ 1015 Lausanne, Switzerland
}

\begin{abstract}
SwissFEL is aiming to produce X-ray pulses from $30 \mathrm{fs}$ down to the attosecond time scale. This requires the compression of the several picosecond long electron bunches produced by a photo-injector to sub-fs level. To achieve this, $40 \mathrm{fs}$ accurate injection of the electron bunches into the main linear accelerator is necessary. Therefore high timing accuracy is required from the drive laser of the electron gun. Furthermore fs scan capability is foreseen for the experimental stations of the FEL. The ultra-short pulse pump-probe lasers therefore need to exhibit outstanding, below $10 \mathrm{fs}$ short term jitter relative to the X-rays. Timing tools for both the electron gun laser and for the experiments are developed. The former is based on electro-optical modulation of the optical reference at $1560 \mathrm{~nm}$ by a signal produced from the gun laser at $260 \mathrm{~nm}$, a concept similar to beam arrival monitors in the linear accelerator, with an expected resolution below $20 \mathrm{fs}$. The latter will use spectrally resolved cross-correlation technique to determine relative jitter between the optical reference and the laser used at the experiments at $800 \mathrm{~nm}$, with fs resolution. These systems will be complemented by electron and X-ray timing tools. In this paper we present the general concept for the laser arrival time measurement and correction, with first results obtained on a Ti:sapphire chirped pulse amplifier system. Shot to shot, short term jitter and long term timing drift measurements are presented, with discussion on the sources of the noise. Plans for the feedback stabilization and the resolution and limitation of the systems are also covered.
\end{abstract}

Keywords: Laser arrival time, synchronization, pump-probe

\section{INTRODUCTION}

X-ray FELs (free electron lasers) produce highly coherent radiation with short pulse duration down to sub-fs, providing ultrahigh brightness for diverse experiments in physics, molecular dynamics, material research and medicine. SwissFEL ${ }^{1}$ is under construction for this purpose and will become operational at the beginning of 2017. It will produce X-ray pulses covering the wavelength range from $1 \AA$ up to $70 \AA$, between $0.3-20$ fs pulse length.

The resolution of the planned pump-probe experiments ${ }^{2,3}$ will depend both on the timing accuracy of the gun laser, which produces the electrons at the injector of the FEL, and on the laser used for the experiments. The parameters for the main laser systems are summarized in Table 1 . The optical reference will be distributed along the $0.74 \mathrm{~km}$ long facility through stabilized links and key requirements are $<10 \mathrm{fs}$ rms jitter and temporal drift stability of $10 \mathrm{fs}$ (peak to peak) ${ }^{4}$. The gun laser will consist of a direct diode pumped $\mathrm{Yb}: \mathrm{CaF}_{2}$ sub-ps laser system converted to its $4^{\text {th }}$ harmonic wavelength and is currently under development ${ }^{5}$. The specification for jitter and long term drift is $<40 \mathrm{fs}$ rms, which is calculated from the performance tolerances for the $\mathrm{FEL}^{6}$. This requires passive stabilization for the short term jitter and active control for the long term drifts. The system used for the experiments will be based on Ti:sapphire laser, delivering a total of $20 \mathrm{~mJ}$ with $30 \mathrm{fs}$ pulses ${ }^{2,3}$. The jitter specification is $150 \mathrm{fs}$ rms both short and long term. Although binning techniques, measuring relative jitter between the laser and the X-ray pulses, are planned to be implemented in SwissFEL ${ }^{7-10}$, the overall jitter performance of the pump laser will ultimately limit the online resolution. Therefore a measurement system with $<10 \mathrm{fs}$ resolution is planned to use as a feedback.

In this paper we present the overall concept for the laser jitter measurement for the SwissFEL facility and show the measurements performed, using an optical cross-correlator to characterize the timing jitter of a Ti:sapphire laser, similar to that, which will be used for SwissFEL experiments.

*marta.divall@psi.ch,phone: +41563102139, fax: +41563102199,www.psi.ch

Advances in X-ray Free-Electron Lasers Instrumentation III, edited by Sandra G. Biedron Proc. of SPIE Vol. 9512, 95121T · ( 2015 SPIE · CCC code: 0277-786X/15/\$18 doi: $10.1117 / 12.2179016$

Proc. of SPIE Vol. $951295121 \mathrm{~T}-1$ 
Table 1.: Laser specifications for the electron gun, the experimental laser and the reference optical master oscillator. In brackets the future aim.

\begin{tabular}{|llll|}
\hline Parameter & $\begin{array}{l}\text { Experiment } \\
\text { Laser }\end{array}$ & $\begin{array}{l}\text { Gun } \\
\text { Laser }\end{array}$ & Units \\
\hline Wavelength & 800 & 260 & $\mathrm{~nm}$ \\
Meas. resolution & $10(1)$ & 25 & $\mathrm{fs}$ \\
$\begin{array}{l}\text { Overall rms jitter } \\
\text { (int. 10Hz to 10MHz) }\end{array}$ & 150 & 40 & $\mathrm{fs}$ \\
Pulse length & 0.03 & $4-10$ & $\mathrm{ps}$ \\
Rise and fall-time & 10 & 700 & $\mathrm{fs}$ \\
Pulse Energy & 10 & 2 & $\mathrm{~mJ}$ \\
\hline Reference wavelength & & 1560 & $\mathrm{~nm}$ \\
Reference pulse length & & 180 & $\mathrm{fs}$ \\
Reference pulse energy & & 0.2 & $\mathrm{~nJ}$ \\
\hline
\end{tabular}

\section{LASER ARRIVAL MONITOR CONCEPT}

The front-end oscillators of the gun and both experiment lasers will be locked to the reference laser that is distributed over stabilized fiber links. However, the rest of the laser chain (stretcher, amplification, compression, wavelength conversion and all the beam paths in-between) may see an increase in timing jitter due to vibration or timing drift due to changes in environmental parameters including temperature, pressure, and humidity, as well as variation in the mains power voltage and frequency. The LAMs (laser arrival time monitors) will provide accurate measurement of jitter and drift along the laser chains. This information will be used for correlation measurements to identify major contributors and thus allowing to passively stabilizing the laser system. To further minimize jitter and drift actively, actuation on the beam path length by continuously variable optical delay stage will be used.

\section{General concept}

Fig. 1 shows an overview of the systems involved for laser jitter measurement and control. The laser oscillators will be locked to the fiber distributed optical reference via RF electronics based locking as well as balanced optical crosscorrelation technique to provide few fs integrated jitter capability ${ }^{11}$. Both systems are developed at PSI in the 'Timing and synchronization' group.

To engage the high resolution LAMs, temporal pulse overlap of the reference and the gun or experiment laser is required. This is achieved by measuring the relative pulse timing in an all-electronic digital arrival time monitor with large range (ns') and ps accuracy. It is based on fast photodiodes and a very high sampling rate ADC (IOXOS). Its output signal is used to actuate on a continuously variable optical delay to establish pulse overlap for the high resolution LAMs. The electronic arrival monitor and the adjustable optical delay are essential, as the configuration of the experiment laser system will change for each user configuration. A spectrally resolved cross-correlation in the case of the experimental laser and electro-optic modulator based measurement system for the gun laser at the cathode will act as a LAM. 


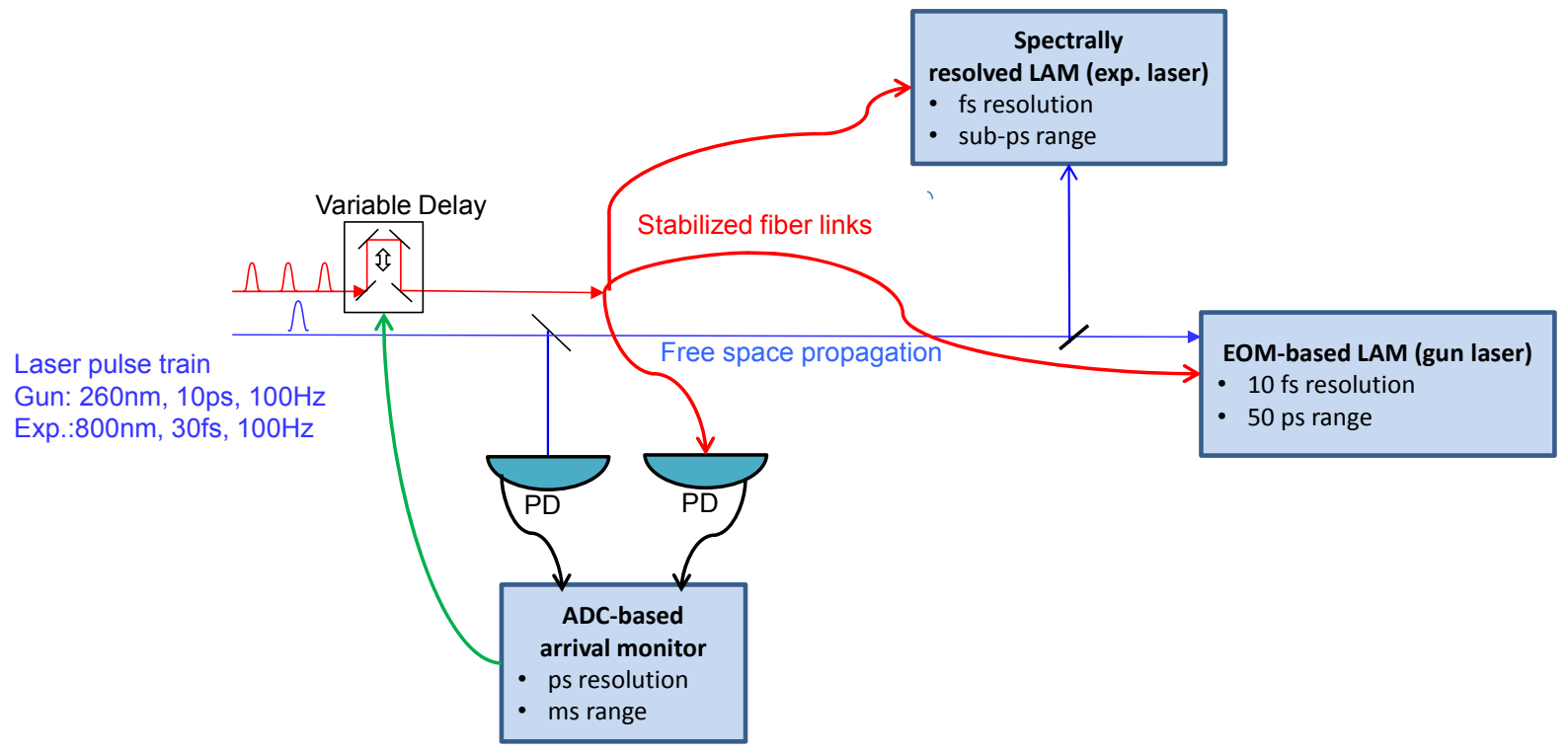

Figure 1. Overview of the systems involved for laser jitter measurement and control

Using the time signal from the electronic arrival time measurement, the variable optical delay line will be actuated to keep optimal signal overlap ensuring best operation of the high resolution LAMs and BOXC.

\section{LAM based on electro-optic modulator}

The EOM-based LAM uses a similar principle to the beam arrival monitors (BAM) developed and tested at PSI ${ }^{12}$. By means of an electro-optic modulator (EOM) timing information is encoded onto the amplitude of the reference pulse train that is then read out by a fast ADC (Fig. 2 a). The modulation signal is the output of a high-speed UV photo diode and filtered and amplified to drive the EOM. Clock and trigger for the ADC are derived from the reference pulse train. The amplitude variation corresponds to the arrival time (Fig. 2 b). Evident is the linear dynamic range limited by the pulse overlap and the slope of the so called s-curve given by the pulse widths. The non-zero average signal level is best suited only for single ended input range ADCs. Using a voltage level shifter or a balanced scheme the full input range of a differential input ADCs can be employed.

Selection and operation of the photodiodes, filters, amplifiers and the EOM is most crucial as not to introduce additional phase shifts and only minimal noise. All LAM components are temperature controlled and the EOM working point is stabilized via a bias voltage controller. Still, optical bandwidth limit may compromise transmitted reference pulses. The detection of the laser (or other) pulses is in principle possible over the entire electromagnetic spectrum, provided that suitable detectors are available. First results will be reported elsewhere.

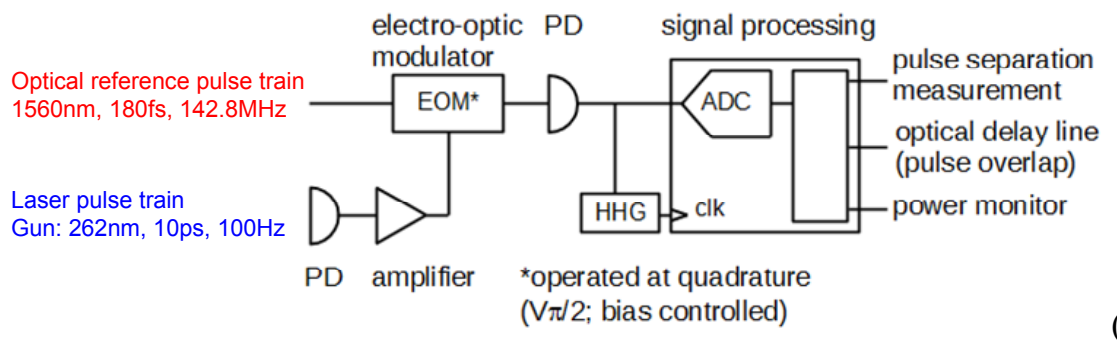

(a) 


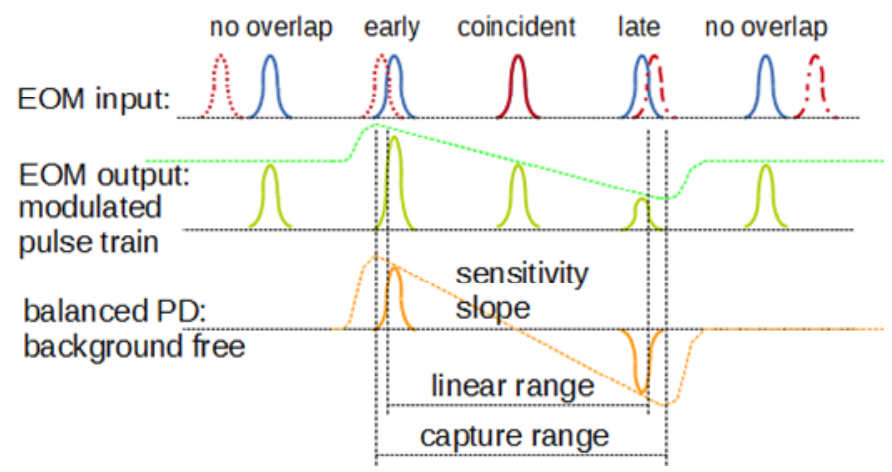

(b)

Figure 2. (a): Simplified schematic of the EOM based LAM setup. (b): Generated signals at different temporal coincidences of the reference and laser pulses.

\section{Spectrally resolved cross-correlator}

The spectrally resolved optical cross-correlation technique is already applied on large OPCPA laser systems to synchronize the pump and the seed laser ${ }^{13-15}$. The optical reference pulse and a few $\mu \mathrm{J}$ of the main laser pulse will be mixed in a non-linear crystal via DFG (difference frequency generation). Scanning the two pulses over each other and measuring the amplitude of the mixed signal provides a simple cross-correlation signal. To spectrally resolve the measurement and therefore remove the effect of amplitude fluctuations, the short pulse from the experimental laser will be stretched before mixing. In this case, the relative delay between the two signals will exhibit in a shift of frequency of the generated DFG signal and can be detected by a spectrometer (Fig. 3). Simulations in Lab2 ${ }^{16}$ show that sub-fs resolution is achievable, using a home-built spectrometer, based on a standard line-detector or CCD camera. Initial results will be presented in the following section.

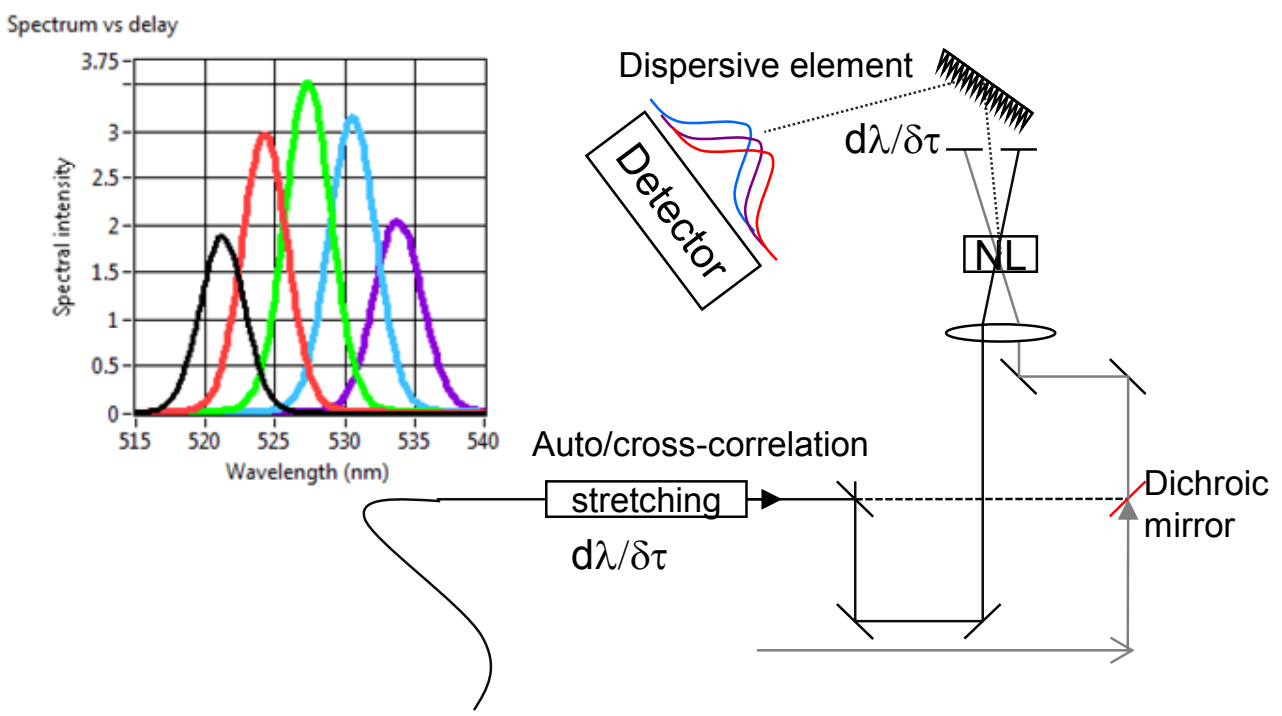

Figure 3. The schema for the spectrally resolved cross-correlation set-up, to be used with a stretched amplifier pulse and the optical reference at $1560 \mathrm{~nm}$. The inset shows the mixed spectrum for different delays, calculated with Lab2. The setup can also be used as an auto-correlator for the stretched pulse. 


\section{MEASUREMENT RESULTS}

\section{The laser}

Currently a Ti:sapphire-based CPA system is available for tests at the former SwissFEL tests injector. This laser is similar to the one, which will be used at the experimental stations ${ }^{17,18}$. The oscillator (Rainbow from Femtolaser) runs at 83.247 MHz and is synchronized via an RF loop. It delivers $\sim 7 \mathrm{fs}$ pulses. The measurements to tests the capability of the spectrally resolved setup were performed with the output of the laser chain after the compressor operating at $100 \mathrm{~Hz}$, taking a few $\mu \mathrm{J}$ level beam. Here the transform limited pulses are $45 \mathrm{fs}$ long. The cross-correlation measurements, to determine long term timing drift of the laser system, were performed using a $5 \mathrm{~mW}$ tap from the oscillator and mixing it with the same compressed output. Timing jitter measurements performed with the UV output of this laser directly at the photo-injector of the SwissFEL Test Facility are reported in Ref. 19.

\section{The setup}

Figure 4 shows the setup. When the polarizing beam splitter is removed two beams from the two parts of the laser system are combined, while in the other case the splitter allows the same beam to be distributed between the two arms. The signals are combined collinearly on the BBO crystal. The non-collinear arrangement allows to spatially filter the second harmonic light produced from each arm before the light is dispersed onto the CCD camera (Basler). The single beam configuration, with a $5 \mathrm{~cm}$ long $\mathrm{CaF} 2$ block used in double path, allowed us to perform resolution and stability measurements on the setup itself, while to two beam configuration made it possible to measure overall jitter and drift from the laser system.
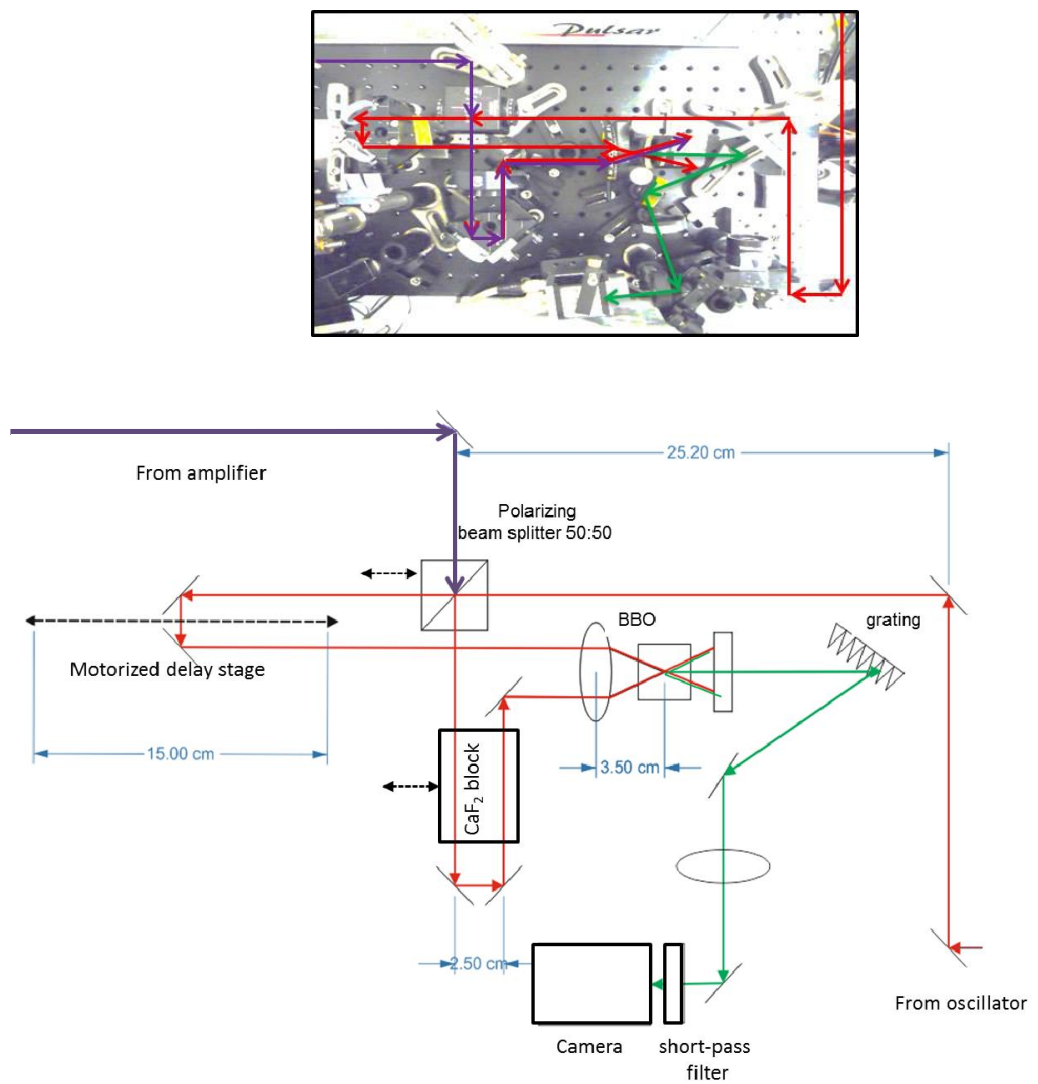

Fig.4.The schematic of the setup. Top: photo with input beams indicated, bottom: diagram of the setup with the removable parts $\left(\mathrm{CaF}_{2}\right.$ block and beam splitter) 
The camera images were visualized on an EPICS based medm panel, where trigger, exposure and region of interest (ROI) can be set and background image can be recorded. For the spectrally resolved measurements a polynomial fit is applied to the spectrum and the peak position is read out as a function of the applied delay between the arms. This helps to increase the total scan region, as even spectra, which are at the edge of the camera chip can still be analyzed. For the cross-correlation measurements an integral is performed over the pixels within the ROI.

\section{Spectrally resolved measurements}

The compressor beam was split in between two arms of the setup and one arm stretched by the $\mathrm{CaF}_{2}$ block. The stretched pulse has a FWHM of $144 \mathrm{fs}$. For the mixing a $0.35 \mathrm{~mm}$ long type I BBO crystal was used, cut for SHG at $800 \mathrm{~nm}$ $(\Theta=29.2 \mathrm{deg})$, anti-reflection coated for $800 \mathrm{~nm}$ on the front surface and $400 \mathrm{~nm}$ on the back surface. The UV grating used, has a groove density of 2400 grooves $/ \mathrm{mm}$. The first order reflection was monitored by the camera.

Figure 5 shows the calibration curve taken with a second order fit applied for maximum accuracy. In the region between -80 and $-180 \mathrm{fs}$ the deviation from the fit is $<1 \mathrm{fs}$. The rms noise of the measurements for each point calculated over 10 points is $\sim 0.33 \mathrm{fs}$, which show the sub-fs resolution capability of the device.
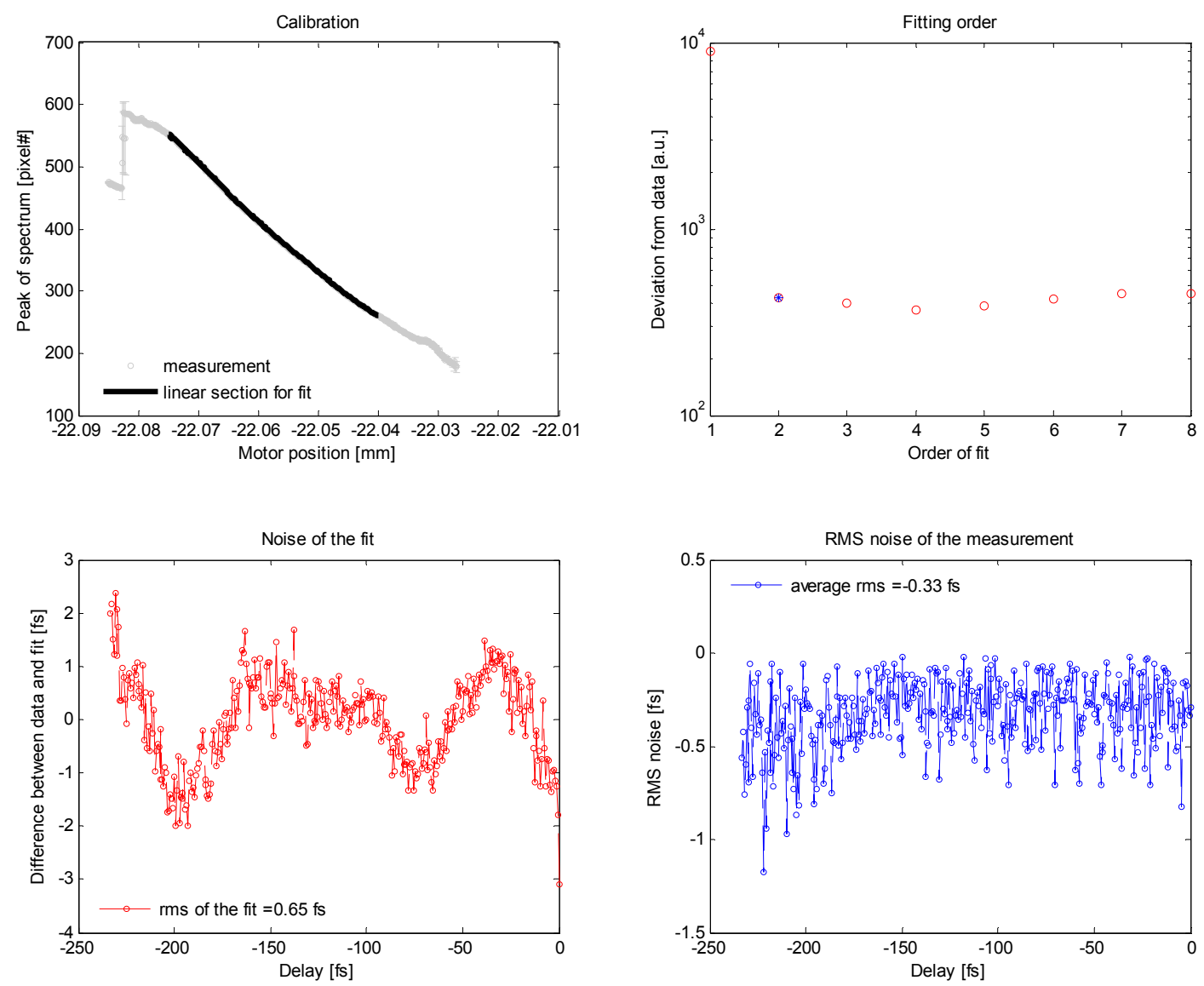

Fig.5. Calibration scan (top left) taken with the beam at the output of compressor, one arm stretched by the CaF $\mathrm{F}_{2}$ block. Second order fit chosen for accuracy (top right) with the error of the fit (bottom left) and the rms noise of the measurement over the delay range (bottom right)

Long term stability of the setup was measured at a fixed delay at the middle of the linear range over 5 hours and is shown in figure 6 . The rms jitter was $0.42 \mathrm{fs}$ with the main contribution coming from drifts related to temperature 
changes in the laser lab. The correlation slope shows a $15 \mathrm{fs} /{ }^{\circ} \mathrm{C}$ drift. In SwissFEL the room will be stabilized to $0.1{ }^{\circ} \mathrm{C}$ accuracy and the temperature of the LAM box will be monitored with a few $\mathrm{mK}$ precision. The system should provide an excellent stability and resolution over time, well within the specification.
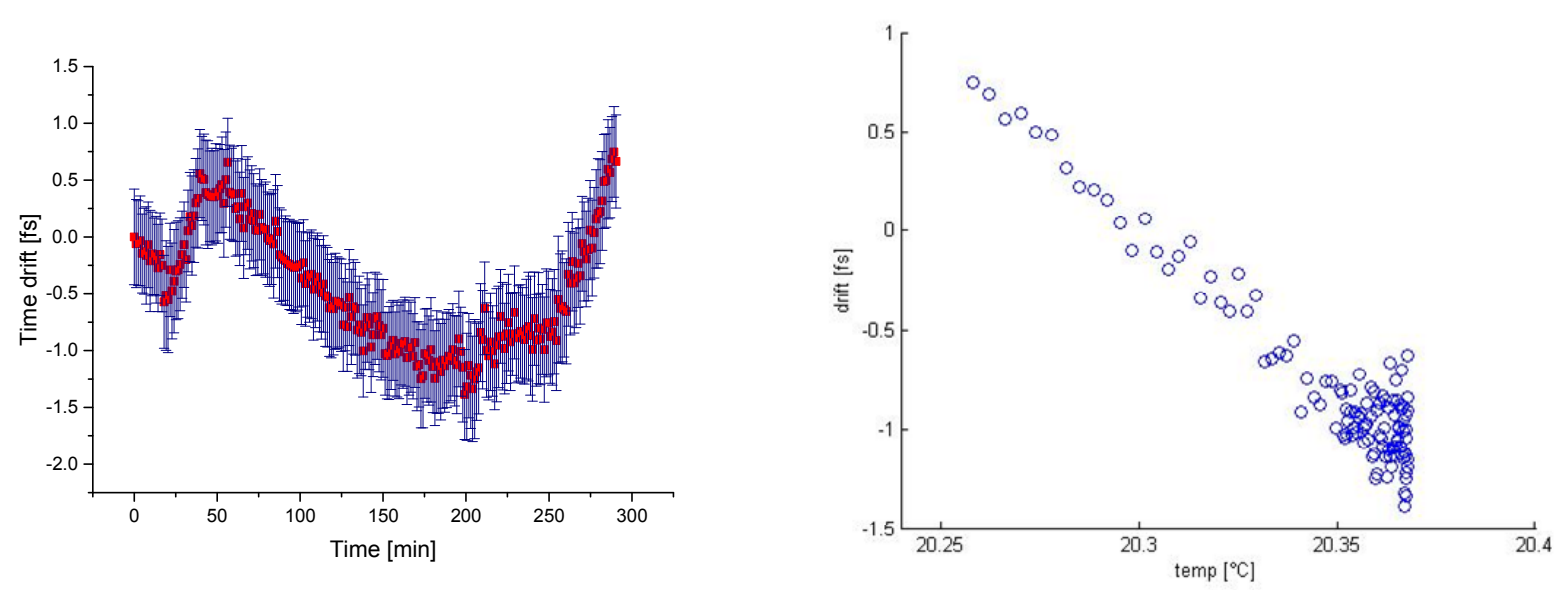

Fig.6. Long term stability measurement of the setup over 5 hours at fixed delay (left) with the drift correlated to the laser room temperature change (right), giving an average rms jitter of $0.42 \mathrm{fs}$ and a drift slope of $15 \mathrm{fs} /{ }^{\circ} \mathrm{C}$

\section{Measurements of the laser system jitter between the oscillator and the amplifier}

The output of the compressor after a total propagation path in the laser system (booster, stretcher, regenerative amplifier, two multi-pass amplifiers) corresponding to $\sim 60 \mathrm{~m}$ was compared to the nearest coinciding oscillator pulse, which was transported to the output of the compressor over a $5 \mathrm{~m}$ stable, enclosed transfer line. The laser was synchronized to an external reference with an excellent $\sim 30 \mathrm{fs}$ integrated jitter $(10 \mathrm{~Hz}-10 \mathrm{MHz})$. The available power from the oscillator makes spectrally resolved measurements hampered by bad signal to noise ratio. Therefore for the long term measurements the grating was replaced by a mirror and the mixed signal was loosely focused onto the camera. Furthermore the crystal was replaced by a $2 \mathrm{~mm}$ long BBO and the amplifier beam was collimated through the crystal to increase the overlapping area between the two beams. The signal was projected onto the camera and the integral of the beam was measured over the selected region of interest. Figure 7 shows the linear section of the cross-correlation, used for the jitter measurements. This time a 4th order fit was chosen for the best accuracy. The resolution and noise of the system is in the few fs range, which is larger than for the spectrally resolved configuration, but is still within the specification and allows for long term drift measurements. The linear range covers $70 \mathrm{fs}$.

Short term jitter measurement is shown on Fig. 8 was performed over 3.5 minutes. The peak to peak jitter over this period was $\sim 30.6 \mathrm{fs}$, while the rms was $\sim 5.6 \mathrm{fs}$.

As the rage of the measurement is $70 \mathrm{fs}$ a slow ON/OFF type feedback was applied, which moved the motor by a welldefined discrete step each time the drift was more than $35 \mathrm{fs}$ in either direction. This has allowed long term measurements over several hours, as shown on Fig.9. Using this simple loop the overall rms jitter was kept below $2.6 \mathrm{fs}$, while to peak to peak deviation was $\sim 20.6 \mathrm{fs}$. The total drift without correction would have been $\sim 150 \mathrm{fs}$ over 1 hour. 

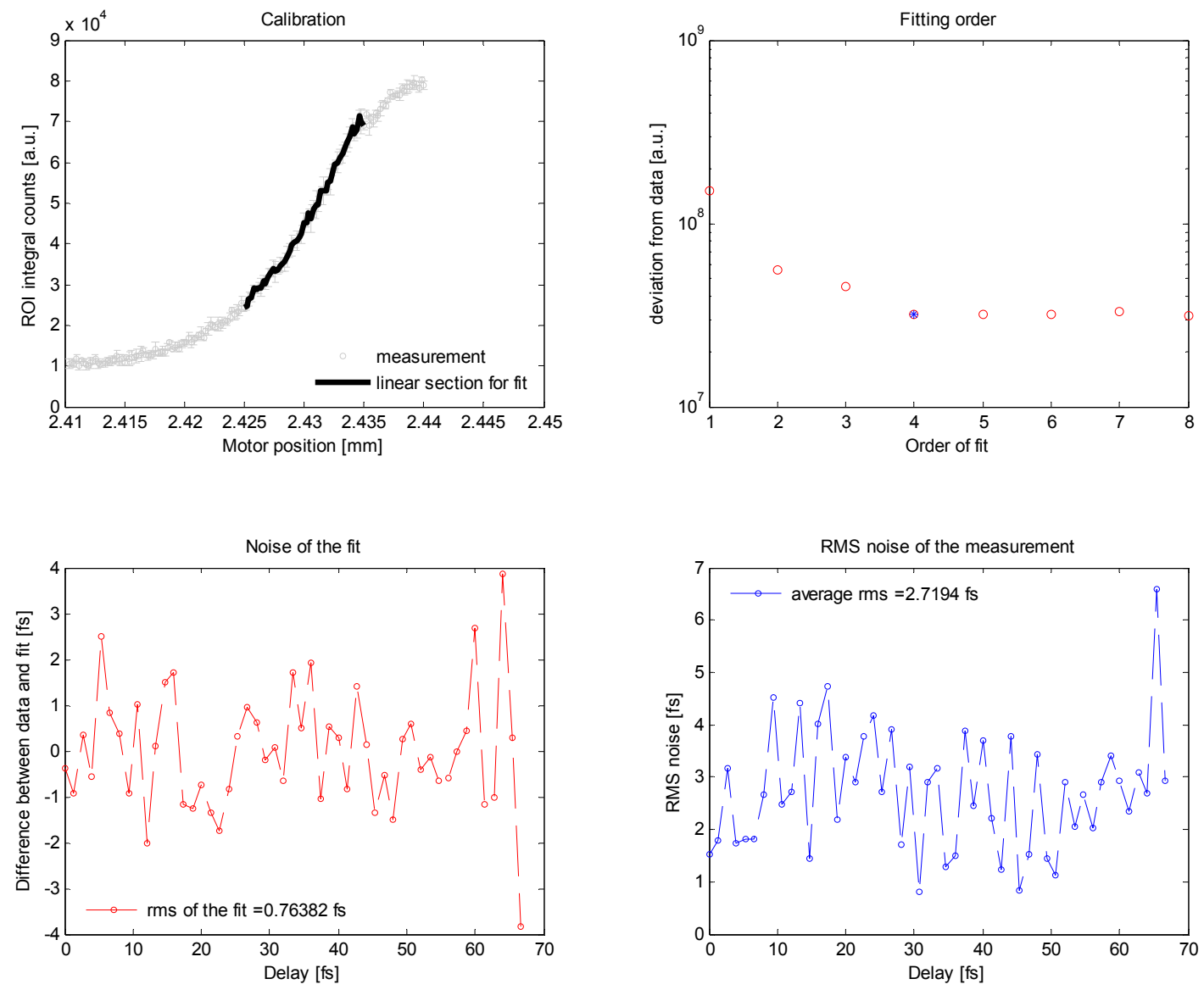

Fig.7. The linear part of the cross-correlation signal (left top), the order of the fit chosen for accuracy (top right) with the error of the fit (bottom left) and the rms noise of the measurement over the delay range (bottom right)

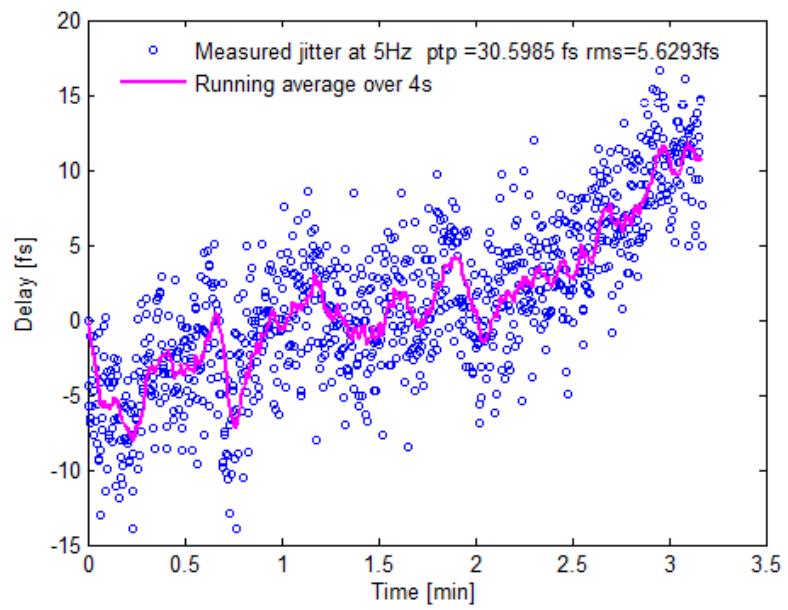

Fig.8. Short term jitter of the laser system, measured at $5 \mathrm{~Hz}$, using the seed oscillator as a reference 

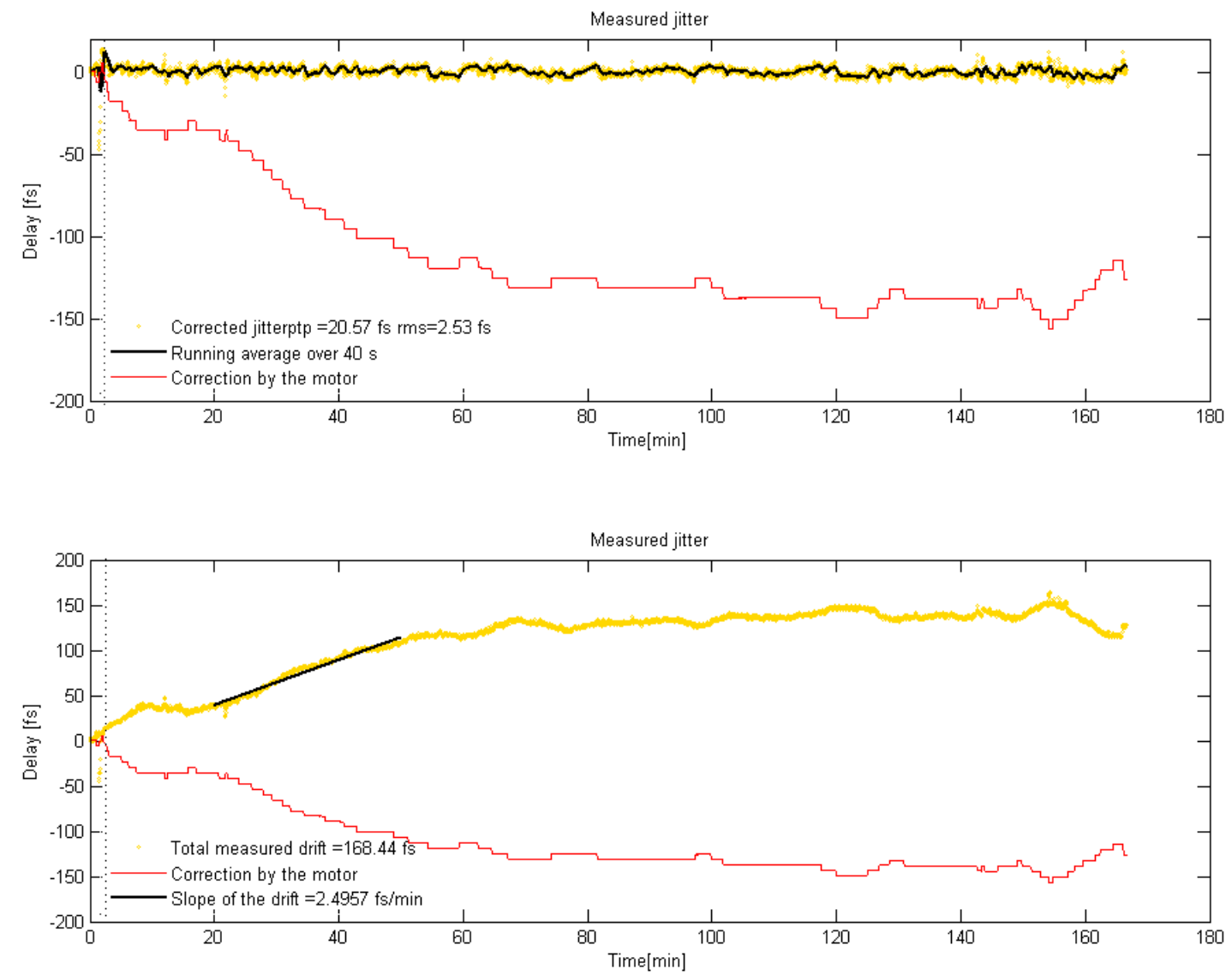

Fig.9. Long term measurement performed with the cross-correlator, using a simple ON/OFF loop to keep the overlap between the pulses. The dashed line marks the time when the loop was turned on.

\section{CONCLUSIONS}

Two different complementing LAM concepts are currently being explored for SwissFEL, i.e. an EOM-based approach for the gun laser and a spectrally resolved optical cross-correlator for the experiment laser. As high resolution LAMs are generally limited in range, therefore an all-electronic coarse arrival time system (ADC-based) and variable optical delay are needed to achieve signal overlap and also to increase dynamic range. Timing information from both LAMs will be used to compensate for drift between the lasers and the machine during machine operation. The LAMs are also used to characterize the laser systems and to find sources of drift and jitter. Initial results show that requirements for resolution and range are fulfilled for the experimental lasers. The developed cross-correlator shows below fs resolution for the spectrally resolved case and a few fs resolution for the intensity cross-correlation case. Initial tests were performed with the existing Ti:sapphire laser system and with a single ON/OFF feedback concept to keep signal overlap. This device will allow for longer term correlation measurements, which in turn will help to find and eliminate long term drift sources. The device can also be used to characterize long transport lines, which will be needed for SwissFEL. 


\section{ACKNOWLEDGMENTS}

The authors would like to thank the laser team (A. Trisorio and C. Vicario) for their support with the Ti:sapphire system. We would also like to thank H. Brands for the camera and E. Divall for the EPICS interface development.

\section{REFERENCES}

[1] SwissFEL, http://www.psi.ch/swissfel/

[2] Christopher J. Milne (ed.) Experimental Station A: Conceptual Design Report http://www.psi.ch/swissfel/CurrentSwissFELPublicationsEN/ESA CDR v4 with coverpage \%282\%29-VM16.pdf

[3] Gerhard Ingold and Paul Beaud (ed.) Conceptual Design Report SwissFEL ARAMIS Endstation ES-B http://www.psi.ch/swissfel/CurrentSwissFELPublicationsEN/ES-B_CDR_2013-0621 v2 with coverpage \%282\%29-VM16.pdf

[4] S. Hunziker, et al., "Reference distribution and synchronization systems for SwissFEL: Concept and First results," IBIC, MOCZB, Monterey, Ca., USA, (2014)

[5] A. Trisorio, M. Divall, C. Vicario, A. Courjaud and C. P. Hauri New concept for the SwissFEL gun laser Proceedings of FEL2013, TUPSO88, New York, NY, USA (2013)

[6] B. Beutner, S. Reiche Sensitivity and tolerance study for the SwissFEL Proc FEL2010 WEPB17, Malmö, Sweden (2010)

[7] Cavalieri, A. L. et al. Clocking femtosecond X-rays. Phys. Rev. Lett. 94, 114801 (2005).

[8] Bionta, M. R. et al. Spectral encoding of X-ray/optical relative delay. Opt. Express 19, 21855-21865 (2011).

[9] Harmand, M. et al. Achieving few-femtosecond time-sorting at hard X-ray free-electron lasers. Nat. Photon. 7, 215$218(2013)$

[10] P.N. Juranić, et al. A scheme for a shot-to-shot, femtosecond-resolved pulse length and arrival time measurement of free electron laser x-ray pulses that overcomes the time jitter problem between the FEL and the laser JINST 9 P03006 (2014)

[11] V. Arsov et al. Optical synchronization of the SwissFEL 250MeV test injector gun laser with the optical master oscillator Proc. FEL2011, TUPA21, Shanghai, China (2011)

[12] V. Arsov, M. Aiba, M. Dehler, F. Frei, S. Hunziker, M. Kaiser, A. Romann,V. Schlott Commissioning and results from the bunch arrival-time monitor downstream the bunch compressor at the SwissFEL injector test facility Proceedings of FEL2014, Basel, Switzerland THP085

[13] Miura et al, Timing stabilized regenerative amplifier with spectral-resolved cross-correlation technique OSA TOPS vol.50 ASSL, 517-521 (2001)

[14]H. Zeng et al., Generation of accurately synchronized pump source for optical parametric chirped pulse amplification, Appl. Phys. B 79, 837-839 (2004)

[15]Z. Major et al. Basic concepts and current status of the petawatt field synthesizer - a new approach to ultrahigh field generation, Laser Rev., vol. 37, 431-436 (2009)

[16]B. Schmidt, M. Hacker, G. Stobrawa, T. Feurer; LAB2-A virtual femtosecond laser lab, http://www.lab2.de

[17] A. Trisorio et al. Ultrabroadband TW-class Ti:Sapphire laser system with adjustable central wavelength, bandwidth and multi-color operation Opt. Exp. 19, 21, 20128-20140 (2011)

[18] C.P. Hauri et al., Wavelength-tuneable UV laser for electron beam generation with low intrinsic emittance Proc. IPAC10 WEPD052, Kyoto, Japan (2010)

[19] M. Csatari Divall, M. Kaiser, S. Hunziker, C. Vicario, B. Beutner, T. Schietinger, M. Lüthi, M. Pedrozzi, and C. P. Hauri, Nucl. Instrum. Methods Phys. Res., Sect. A 735, 471 (2014) 